\title{
Numerical Simulation of Film Cooling in Supersonic Flow
}

\author{
Johannes M. F. Peter and Markus J. Kloker
}

\begin{abstract}
High-order direct numerical simulations of film cooling by tangentially blowing cool helium at supersonic speeds into a hot turbulent boundary-layer flow of steam (gaseous $\mathrm{H}_{2} \mathrm{O}$ ) at a free stream Mach number of 3.3 are presented. The stagnation temperature of the hot gas is much larger than that of the coolant flow, which is injected from a vertical slot of height $s$ in a backward-facing step. The influence of the coolant mass flow rate is investigated by varying the blowing ratio $F$ or the injection height $s$ at kept cooling-gas temperature and Mach number. A variation of the coolant Mach number shows no significant influence. In the canonical baseline cases all walls are treated as adiabatic, and the investigation of a strongly cooled wall up to the blowing position, resembling regenerative wall cooling present in a rocket engine, shows a strong influence on the flow field. No significant influence of the lip thickness on the cooling performance is found. Cooling correlations are examined, and a cooling-effectiveness comparison between tangential and wall-normal blowing is performed.
\end{abstract}

\section{Introduction}

Film cooling by injection of a cold secondary gas in a hot-gas main flow is an effective method to provide thermal protection of solid surfaces, for example for the nozzle extension of advanced rocket engines. The coolant can be injected either in wallnormal fashion through holes/slits or tangentially to the wall through a backward facing step. Among the first studies of film cooling under supersonic conditions is the work of Goldstein et al., who experimentally investigated tangential blowing of air and helium and wall-normal blowing of air into a laminar air flow at a Mach

J. M. F. Peter · M. J. Kloker $(\bowtie)$

Institute of Aerodynamics and Gas Dynamics, University of Stuttgart,

Pfaffenwaldring 21, 70569 Stuttgart, Germany

e-mail: markus.kloker@iag.uni-stuttgart.de

J. M. F. Peter

e-mail: johannes.peter@iag.uni-stuttgart.de

(C) The Author(s) 2021

N. A. Adams et al. (eds.), Future Space-Transport-System Components

under High Thermal and Mechanical Loads, Notes on Numerical Fluid Mechanics

and Multidisciplinary Design 146, https://doi.org/10.1007/978-3-030-53847-7_5 
number of 3 . They proposed various formulas to correlate their data [2, 3]. Hombsch and Olivier used a shock tunnel to study slot-, hole- and step-injected film cooling with laminar and turbulent main flow [5]. Juhany et al. performed experimental investigations on tangential film injection of air and helium to study the cooling performance and shock/cooling-film interaction [6] while Konopka et al. [13] used large-eddy simulations for the same problem. Song and Shen experimentally studied the effect of feeding pressure [22] and Mach number [23] on the flow-field structure in supersonic film-cooling with tangential blowing using schlieren imaging, but did not measure the wall heat flux.

In this work high-order direct numerical simulations (DNS) are employed for fundamental investigations of the cooling of supersonic boundary-layer flows. In a first study campaign a laminar hot main flow at a Mach number of about 2.7 and wall-normal blowing through slits or hole arrays has been investigated. The cooling effectiveness of wall-normal injection is, for few orifices at the wall, smaller than for tangential blowing through a backward facing step, but the resulting coolinggas film near the wall can be more easily renewed by repeated injection. The DNS, employing a highly accurate time-stepping scheme, allow to identify situations where the steady laminar flow state is destabilized by the cool blowing, strongly degrading the cooling effectiveness by invoked turbulence. The injection of cool gas translates into a film of coolant gas at the wall, reducing both the temperature difference and the mean wall shear stress by lowering the viscosity and the velocity gradient in the blowing region, and can best be realized by spanwise slits or micro-holes. For effusion cooling through non-small discrete holes the alteration of the local wall shear is of importance, due to the induced vortex structures. Here regions of enhanced wall shear exist, increasing locally the heat load at and downstream of the hole sides, by high-speed streaks. The effect is most pronounced for a very cool wall like in shortduration shock-tunnel experiments, but is much weaker for a radiative-adiabatic wall as present in thermal-equilibrium situations [14]. Simple blowing modelling by fixing the blowing distribution at the wall in fast CFD tools has implications: For narrow placed orifices a standard modelling with no knowledge of the actual blowing distribution resulting from included channels and a plenum chamber is inappropriate and indicates a false, too high critical blowing ratio for inducing turbulence tripping by the blowing [9]. Various cooling gases have been considered for binary-gas flow, and the comparison with the results of analogous experiments at RWTH Aachen showed somewhat lower experimental values, most probably caused by disturbances coming from the blowing device, rendering the flow no more laminar [11]. Employing simulations with deliberately manufactured cooling gases, cooling-gas properties beneficial for a high cooling effectiveness could be clearly identified: The diffusion coefficient shows virtually no influence on the effectiveness, whereas low coolinggas viscosity, low thermal conductivity, high heat capacity, low molar mass and low density turned out to be highly beneficial. Cooling with light gases like helium or hydrogen leads however to a destabilization of the laminar flow, contrary to heavy gases. The blowing-jet penetration height in the hot boundary-layer flow seems to 
play an important role, being higher with a light cooling gas due to the increased blowing velocity at a kept coolant mass flow. An extension of the well-working single-species cooling-effectiveness correlation to binary gas-mixture flows turned out to be challenging, if possible at all; not only the heat capacity or the molar mass have to be taken into account.

The second study campaign was initiated with fundamental investigations on the influence of wall-normal slit blowing into a turbulent air main flow, using air or helium as coolant and neglecting chemical reactions. The DNS results of this study [10] provided valuable benchmark data for the validation of less expensive and more flexible conventional CFD methods using turbulence models. The mixing by turbulence over-compensates the beneficial influence of a fuller mean-flow profile with larger wall shear as known from a favorable streamwise pressure gradient in laminar flow, and a higher cooling-gas mass flux is necessary for the same cooling performance. Turbulence gives rise to a much stronger wall-normal heat conduction compared to the laminar case, resulting in a more rapid heating of the cooling stream. A similar cooling effect is reduced to about $30 \%$ of the laminar streamwise stretch. Moreover, the pressure increase by the blockage effect of the blowing is stronger due to the larger hot-gas velocity close to the wall, and a larger plenum pressure is necessary for the same blowing rate. The simulated and simply modeled blowing setups largely give the same results in the case of the laminar boundary layer, despite there being heat conduction into the channel flow in the simulations including the channel. For the turbulent boundary layer, however, turbulent fluctuations travel into the channel, leading to a premixing process, and thus an effectiveness loss of about $10 \%$ (helium) to $15 \%$ (air). For a more accurate blowing modeling, the prescribed cooling-gas mass fraction, temperature, and turbulence distribution along the slit especially need to be more adapted to the actual profiles computed in this work. Helium blowing leads to a higher cooling effectiveness, mainly due to its high heat capacity. At an equal blowing rate (density times blowing velocity), a light coolinggas jet has higher momentum. This leads to a higher boundary-layer penetration but, due to the lower density, also stronger deflection, and a thicker cooling-film results. The decline of the cooling effectiveness with turbulence is slightly less for helium, despite the main-flow turbulent kinetic energy penetrates deeper into the channel, and the temperature fluctuations are distinctly higher downstream, starting palpably in front of the slit. But, the turbulent kinetic energy is lower in the downstream cooling range of the slit with helium. A small Reynolds-number-lowering effect in the case of helium blowing is present, but it is far too small to cause a relaminarization of the boundary layer.

DNS of transpiration cooling with uniform blowing in a turbulent air boundary layer [1] has shown that the peak turbulent kinetic energy moves away from the wall to the region of the new shear maximum between the low-momentum coolant and the high-momentum hot gas. A derived new model accounts for both heat advection and film accumulation and shows good agreement with the DNS data. Using smaller discrete slits at fixed total coolant flow rate leads to a clear tendency to the uniform blowing case, justifying the use of the latter simple boundary condition. 
In this paper, the complex interaction between a hot turbulent main flow and a coolant gas tangentially injected through a backward facing step is investigated. The physical phenomena governing the flow field, the unavoidable gas mixing process, and thus the wall heat load are scrutinized. Existing film-cooling correlations are examined, and design-guidelines for film-cooling applications and reference cases for turbulence modelling used in faster simulations tools like RANS or LES are prepared.

The paper is organized as follows: The flow setup investigated is described in Sect. 2 and the numerical method used for the DNS is described in Sect. 3. The results from the film-cooling simulations are discussed in Sects. 4 and 5 provides concluding remarks.

\section{Flow Configuration}

The hot flow is superheated steam (gaseous $\mathrm{H}_{2} \mathrm{O}$, i.e. the product of a combustion of hydrogen and oxygen) at a stagnation pressure and temperature of $p_{0}^{\star}=30 \mathrm{bar}$ and $T_{0}^{\star}=3650 \mathrm{~K}$, respectively; the coolant is helium at $T_{0, c}^{\star}=330 \mathrm{~K}$. The hot-flow stagnation conditions are chosen to match the experiments by Ludescher and Olivier [15] (sub-project "Film Cooling in Rocket Nozzle Flows") for a subscale conical nozzle with a detonation tube to generate rocket-engine-like stagnation conditions for a short duration $(\approx 7-10 \mathrm{~ms})$. The nozzle flow has been analyzed using steady-state RANS-simulations of a one-species gas to yield the flow conditions at an expansion ratio of $\varepsilon=14$, for details see [18]. The DNS are performed in a near-wall domain using the results from the RANS analysis. Note that only the free-stream data at the given expansion ratio is used as free-stream condition, whereas the pressure gradient from the experiment is not considered. Also, the constant low wall temperature due to the short-time experiment $\left(T_{w, \exp }^{\star} \approx 330 \mathrm{~K}\right)$ is not matched, rather the film-cooled wall section is always treated adiabatic, whereas the wall up to the blowing position is either treated as adiabatic or isothermal with a wall temperature of $T_{w}^{\star}=1700 \mathrm{~K}$. The resulting parameters for the DNS are listed in Table 1, along with the used thermophysical properties of hot $\mathrm{GH}_{2} \mathrm{O}$ and cold helium.

\subsection{Film Cooling}

Helium is injected supersonically through a 2D spanwise slot opening in a backwardfacing step. The slot has a height of $s^{\star}$ and the lip thickness is $t^{\star}$, see Fig. 1. A parabolic velocity profile is taken for the coolant supply exit flow, according to a laminar flow in the cooling-gas channel. A channel centerline Mach number $M a_{c}$ is chosen and the velocity $u_{c}^{\star}\left(y^{\star}\right)$ is then derived from the total temperature $T_{0, c}^{\star}$. The static-temperature profile is gained from the velocity profile using a total temperature that linearly varies from $T_{0, c}^{\star}$ in the centerline to the coolant recovery temperature 
$T_{\text {rec,c }}^{\star}$ at the channel wall. The static pressure $p_{c}^{\star}$ is taken constant over the slot height and the density $\rho_{c}^{\star}$ is derived from the equation of state. The blowing ratio $F=\left(\rho_{c}^{\star} u_{c}^{\star}\right) /\left(\rho_{\infty}^{\star} u_{\infty}^{\star}\right)$ is varied by varying $p_{c}^{\star}$ (and thus $\rho_{c}^{\star}$ with $\left.\rho_{c}^{\star} \propto p_{c}^{\star}\right)$, leading to different ratios of cooling-gas to free-stream pressure. Note that all reported coolant exit conditions (i.e. pressure-matched, over- or under-expanded) are based on the free-stream pressure, not on the pressure behind the step without a secondary stream. In all presented cases the free stream velocity is higher than the coolant velocity, i.e. the velocity ratio $V=u_{c}^{\star} / u_{\infty}^{\star}<1$ and therefore the flow is core driven. The blowing ratios are reported using the averaged mass flow rate through the slot. Four different step geometries are used in the presented studies, listed in Table 2. Geometry G01 marks the reference configuration, G01a and G01b have the same slot height $s^{\star}$, but a different lip thickness $t^{\star}$, while for G02 the slot height is increased by $50 \%$ at constant lip height. The geometry G01 was chosen as reference case because it resembles the step dimensions in the experiments from Ludescher and Olivier [15].

Table 1 Free-stream conditions for the DNS and thermophysical parameters of superheated steam and helium

\begin{tabular}{l|l|l|l|l|l|l}
\hline \multicolumn{2}{l|}{ Free stream } & & Steam & Helium & \\
\hline$M a_{\infty}$ & 3.3 & & $P r$ & 0.8 & 0.7 & \\
\hline$u_{\infty}^{\star}$ & 3383 & {$[\mathrm{~m} / \mathrm{s}]$} & $\kappa$ & 1.15 & 1.66 & \\
\hline$T_{\infty}^{\star}$ & 1980 & {$[\mathrm{~K}]$} & $R^{\star}$ & 461.5 & 2077.3 & {$[\mathrm{~J} /(\mathrm{kg} \mathrm{K})]$} \\
\hline$p_{\infty}^{\star}$ & 0.28 & {$[\mathrm{bar}]$} & Sutherland $\mu_{r e f}^{\star}$ & $1.12 \cdot 10^{-5}$ & $1.85 \cdot 10^{-5}$ & {$[\mathrm{~kg} /(\mathrm{m} \cdot \mathrm{s})]$} \\
\hline$\rho_{\infty}^{\star}$ & 0.0306 & {$\left[\mathrm{~kg} / \mathrm{m}^{3}\right]$} & Sutherland $C^{\star}$ & 1064.0 & 79.44 & {$[\mathrm{~K}]$} \\
\cline { 4 - 7 } & & & Sutherland $T_{r e f}^{\star}$ & 350.0 & 273.1 & {$[\mathrm{~K}]$} \\
\hline
\end{tabular}

Fig. 1 Detailed view of step region

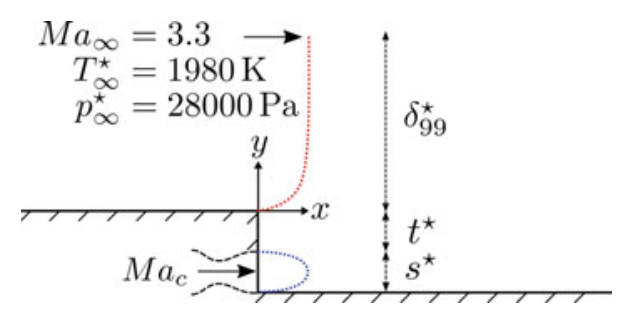

Table 2 Geometries

\begin{tabular}{l|l|l}
\hline Geometry & $t^{\star}(\mathrm{mm})$ & $s^{\star}(\mathrm{mm})$ \\
\hline G01 & 1 & 0.6 \\
\hline G01a & 2 & 0.6 \\
\hline G01b & 0.5 & 0.6 \\
\hline G02 & 1 & 0.9 \\
\hline
\end{tabular}




\section{Numerical Method}

This section is intended to give a brief overview of the simulation setup, extensive details can be found in the referenced literature. For the DNS we use our in-house code NS3D, which has been used successfully for the calculation of film and effusion cooling in laminar and turbulent supersonic boundary-layer flow [7, 9, 11, 14].

The governing equations for a flow of two mixing, non-reacting calorically perfect gases are the continuity equation, the three momentum equations, the energy equation, and the equation of state, all for the mixture values. Additionally, a second continuity equation for one of the gas species is needed, and ordinary and thermal diffusion has to be considered. The equations are non-dimensionalized using the free-stream values of velocity $u_{\infty}^{\star}$, density $\rho_{\infty}^{\star}$, temperature $T_{\infty}^{\star}$, and the pressure is made dimensionless by $\left(\rho_{\infty}^{\star} u_{\infty}^{\star}{ }^{2}\right)$ [11]. The subscript $\infty$ refers to free-stream values while the asterisk ${ }^{\star}$ marks dimensional quantities. Both gas species have constant Prandtl number $\operatorname{Pr}_{i}$ and constant ratio of specific heats $\kappa_{i}=c_{p, i} / c_{v, i}$, where the species number is indicated by the subscript $i$. The equations are solved using a compact finite difference scheme of 6th-order [8] and an explicit 4th-order 4-step Runge-Kutta scheme.

A sketch of the simulation domain is presented in Fig. 2. Extensive details can be found in $[19,20]$ and details about the setup validation can be found in [18]. The length scales are non-dimensionalized by the inlet boundary-layer thickness $\delta_{99, i}^{\star}$. The origin of the coordinate system is at the upper edge of the backward-facing step. Domain size and grid spacing are set to meet the resolution requirements for turbulent flat-plate DNS [21, 27]. At solid walls, the no-slip and no-penetration boundary condition is imposed on the velocity components, $u=v=w=0$. For the adiabatic condition the wall temperature is computed by a 5 th-order one-sided finite difference from $(\partial T / \partial y)_{w}=0$; for an isothermal wall the temperature is set to a fixed value. In both cases the wall pressure is gained like the adiabatic wall temperature from $(\partial p / \partial y)_{w}=0$, and the density is calculated from the equation of

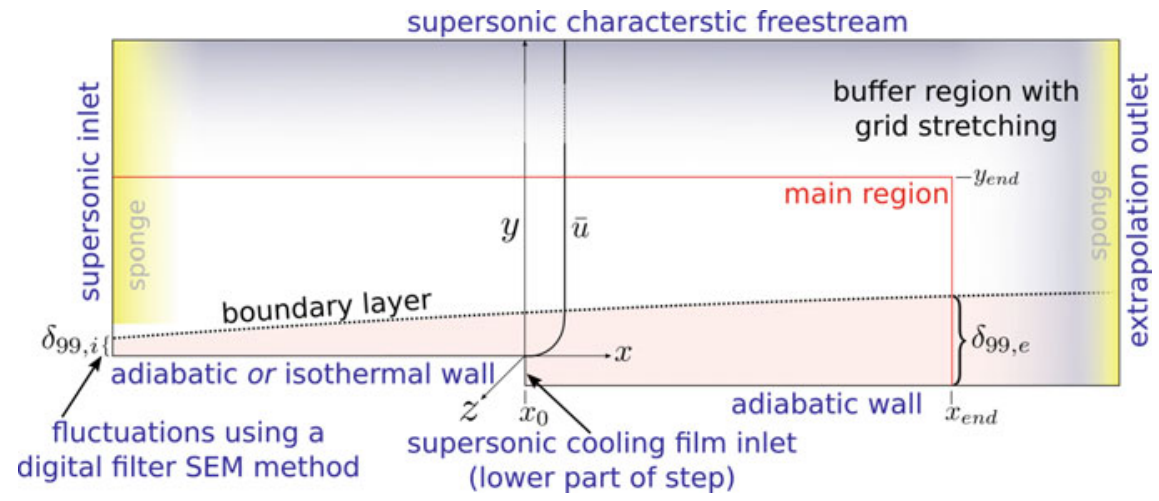

Fig. 2 Setup for the film cooling DNS. The flow is assumed periodic in the z-direction 
state. At the free stream, a spatial supersonic characteristic condition is used. At the outflow, all flow quantities are extrapolated from the field using a 2nd-order parabola. At the main flow inlet a pseudo-turbulent unsteady velocity field is generated using a digital filtering synthetic-eddy method (SEM) [12].

\section{Results}

The effect of the coolant film on the temperature of an adiabatic wall is quantified by the adiabatic cooling effectiveness

$$
\eta_{a d}=\frac{T_{r e c, \infty}-T_{w}}{T_{r e c, \infty}-T_{r e c, c}},
$$

where $T_{r e c, \infty}$ is the hot-gas recovery temperature, $T_{r e c, c}$ is the coolant recovery temperature, and $T_{w}$ is the wall temperature with cooling. We follow the commonly employed naming scheme of Stollery and El-Ehwany [25] to name the different regions of $\eta_{a d}$, see Fig. 3 .

\subsection{Influence of Coolant Mass Flow Rate}

Four different blowing ratios have been simulated to investigate the influence of the coolant mass flow rate. The cases are listed in Table 3. The lowest blowing ratio $F=0.3 \overline{3}$ in case C-I represents an over-expanded flow at the cooling-channel nozzle outlet. For case $\mathrm{C}$-II the pressure is matched at the nozzle exit, resulting in a blowing ratio of $F=0.59,{ }^{1}$ while for the two higher blowing ratios the flow is under-expanded with $F=0.6 \overline{6}$ (case C-III) and $F=1.00$ (case C-IV), respectively. To investigate a possible influence of the slot height an additional case with a blowing ratio of $F=0.6 \overline{6}$ has been simulated where the slot height is increased by $50 \%$ (case CIIIa), which leads to the same non-dimensional coolant mass flow rate $F \cdot s$ as case C-IV. To investigate correlation and scaling formulae the higher slot height has also been simulated with a blowing ratio of $F=1.00$ (case $\mathrm{C}$-IVa). Figure 4 shows the cooling effectiveness $\eta_{a d}$ over the distance to the slot $x^{\star}$ (left) and over the distance scaled using the coolant mass flow rate $x /(F \cdot s)$ ("mass effectiveness", right) for cases $\mathrm{C}$-I to C-IVa. As expected, all cases show the same general behavior with a perfect-cooling region followed by an $x^{-m}$-decay, and higher blowing ratios show a better cooling effectiveness for constant distance to the step. Comparing the cases $\mathrm{C}$ IIIa and C-IV with kept coolant mass-flux, injection through the smaller slot appears beneficial. Both cases show nearly the same decay rate but for case C-IIIa $\eta_{a d}$ deviates somewhat earlier from one. This contradicts the experimental findings of Ludescher

\footnotetext{
${ }^{1}$ Actual $F=0.58644$ for the matched-pressure case C-II.
} 


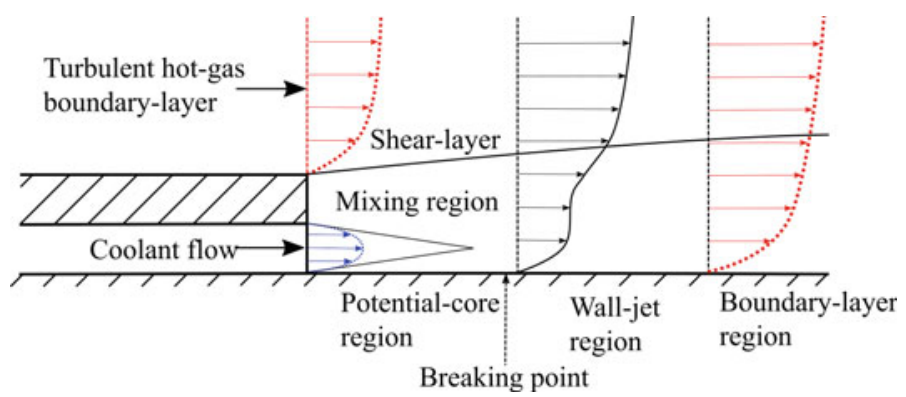

Fig. 3 Main flow characteristics of supersonic film cooling with laminar slot injection [25]

Table 3 Investigated blowing ratios and cooling stream condition

\begin{tabular}{l|l|l|l|l|l|l}
\hline Case & $F$ & Geometry & $s^{\star}(\mathrm{mm})$ & $p_{c}^{\star}(\mathrm{Pa})$ & $p_{c} / p_{\infty}$ & $\begin{array}{l}\text { Coolant exit } \\
\text { condition }\end{array}$ \\
\hline C-I & $0.3 \overline{3}$ & G01 & 0.6 & 15915 & 0.584 & Overexpanded \\
\hline C-II & 0.59 & G01 & 0.6 & 28000 & 1.000 & Matched \\
\hline C-III & $0.6 \overline{6}$ & G01 & 0.6 & 31830 & 1.168 & $\begin{array}{l}\text { (weakly) } \\
\text { underexpanded }\end{array}$ \\
\hline C-IIIa & $0.6 \overline{6}$ & G02 & 0.9 & 31830 & 1.168 & $\begin{array}{l}\text { (weakly) } \\
\text { underexpanded }\end{array}$ \\
\hline C-IV & 1.00 & G01 & 0.6 & 47745 & 1.752 & Underexpanded \\
\hline C-IVa & 1.00 & G02 & 0.9 & 47745 & 1.752 & Underexpanded \\
\hline
\end{tabular}

and Olivier [15], who found that the specific cooling effectiveness increases with increasing slot height. Note that in the experiment the flow changed from underto over-expanded with the slot-height increase while here both cases are underexpanded. Best mass effectiveness for perfect cooling (i.e. longest potential-core region) is found for the matched-pressure case C-II, while in the boundary-layer region up to $x /(F \cdot s) \approx 150$ the mass-specific effectiveness is higher for lower blowing ratios and lower slot heights.

\subsection{Influence of Coolant Mach Number}

The coolant Mach number can be influenced by changing the expansion ratio at the slot opening: either the slot height is changed at kept throat height, or vice-versa. Table 4 shows the cases for the Mach-number investigation. The two baseline cases are C-IIIa and C-IV with a coolant Mach number of $M a_{c}=1.8$. Case C-IIIa-Ma represents a further expansion of the coolant flow with kept throat height compared 

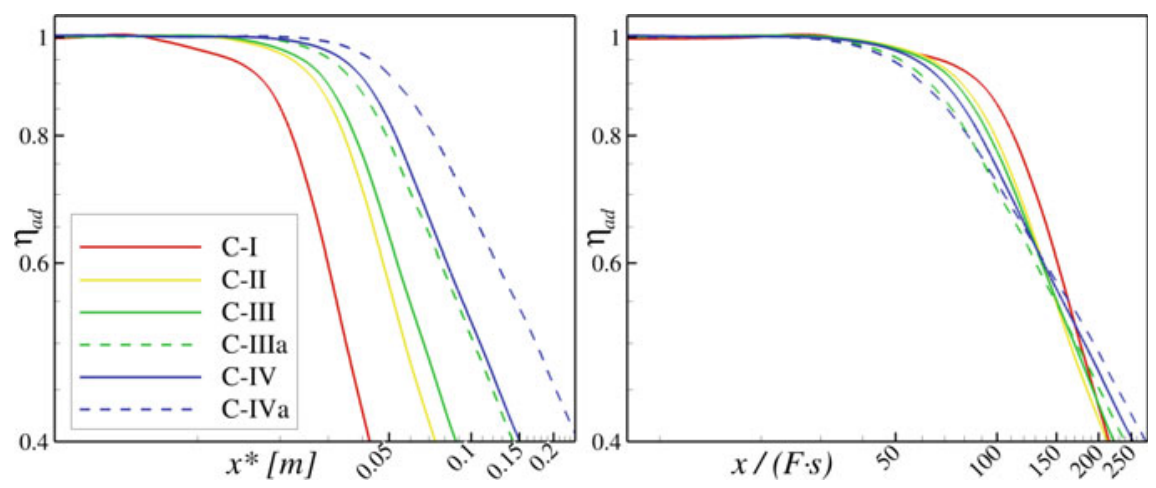

Fig. 4 Comparison of mean cooling effectiveness $\eta_{a d}$ for variation of the coolant mass flow rate

Table 4 Investigated coolant Mach numbers with momentum ratio M

\begin{tabular}{l|l|l|l|l|l|l}
\hline Case & $F$ & Geometry & $s^{\star}(\mathrm{mm})$ & $p_{c}^{\star}(\mathrm{Pa})$ & $M a_{c}$ & $M$ \\
\hline C-IIIa & $0.6 \overline{6}$ & G02 & 0.9 & 31830 & 1.80 & 0.221 \\
\hline C-IIIa-Ma & $0.6 \overline{6}$ & G02 & 0.9 & 22200 & 2.42 & 0.254 \\
\hline C-IV & 1.00 & G01 & 0.6 & 47745 & 1.80 & 0.331 \\
\hline C-IV-Ma & 1.00 & G01 & 0.6 & 32037 & 2.50 & 0.387 \\
\hline
\end{tabular}

Fig. 5 Cooling effectiveness $\eta_{a d}$ for a variation of coolant Mach number

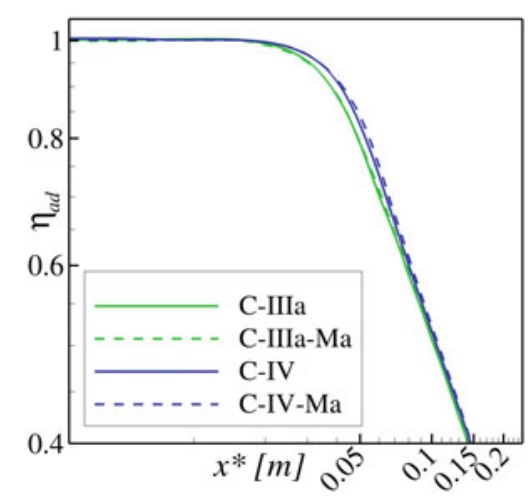

to C-IV, while C-IV-Ma has a smaller (virtual) throat. All four cases have the same coolant mass flow rate $F \cdot s$. A change in the coolant velocity leads to a change in the momentum ratio $M=\left(\rho_{c} u_{c}\right)^{2} /\left(\rho_{\infty} u_{\infty}\right)^{2}$ also listed in Table 4. Note that $M$ is calculated using the average values over the slot height. As is evident from Fig. 5, the present investigation shows no significant influence of the coolant Mach number. 


\subsection{Influence of the Upstream Wall Temperature}

Most of the studies on supersonic film cooling from literature were not performed under realistic rocket-engine-like flow conditions, i.e. the hot-gas temperature and especially the total-temperature ratio between coolant and main flow were not representative of real-world (rocket engine) applications. In a practical application with a hot-gas temperature far above the temperature limit of the used material, the wall up to the blowing position must be cooled, e.g. using regenerative cooling. The assumption of an overall adiabatic wall can only be used as a reference case. The difference between a fully adiabatic case and a case with strong wall cooling upstream of the blowing position, $T_{w}^{\star}=1700 \mathrm{~K}$, at otherwise identical conditions (i.e. the filmcooled wall is adiabatic) is therefore investigated, see Table 5. This leads to ratios of $T_{w}^{\star} / T_{\text {rec }, \infty}^{\star} \approx 0.49$ and $T_{w}^{\star} / T_{\infty}^{\star} \approx 0.86$. The reader is referred to [19] for a discussion of the differences in the oncoming hot-gas boundary layer. As a short summary, the wall cooling leads to a slightly thinner boundary layer with an approximately $7 \%$ higher value of the skin friction coefficient at the blowing location. Figure 6 shows the cooling effectiveness $\eta_{a d}$ along the film-cooled wall. Two main differences are visible: for case C-IV-OC, $\eta_{a d}$ deviates earlier, unexpectedly, from the ideal value and shows a stronger decay up to $x / s=75$, but in the boundary-layer region downstream the pre-cooling leads to the expected lower decay rate and therefore a better cooling effectiveness for $x / s>125$. For both cases a generalized inflection point (GIP), determined from $\frac{\partial}{\partial y}\left(\bar{\rho} \frac{\partial \bar{u}}{\partial y}\right)=0$ [16], exists at $x / s=3$ in the upper-lip shear-layer, indicating strong inviscid instability in the mean flow. The cooling of the upstream

Table 5 Investigated cases for the upstream wall temperature influence

\begin{tabular}{l|l|l|l}
\hline Case & $F$ & Geometry & $\begin{array}{l}\text { Upstream wall temperature } \\
\text { condition }\end{array}$ \\
\hline C-IV & 1.00 & G01 & Adiabatic, $T_{r e c, \infty}^{\star} \approx 3481 \mathrm{~K}$ \\
\hline C-IV-OC & 1.00 & G01 & Isothermal, $T_{w}^{\star}=1700 \mathrm{~K}$ \\
\hline
\end{tabular}

Fig. 6 Comparison of mean adiabatic cooling effectiveness $\eta_{a d}$

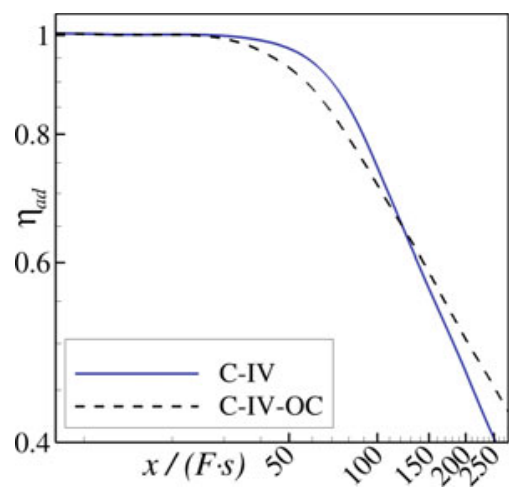




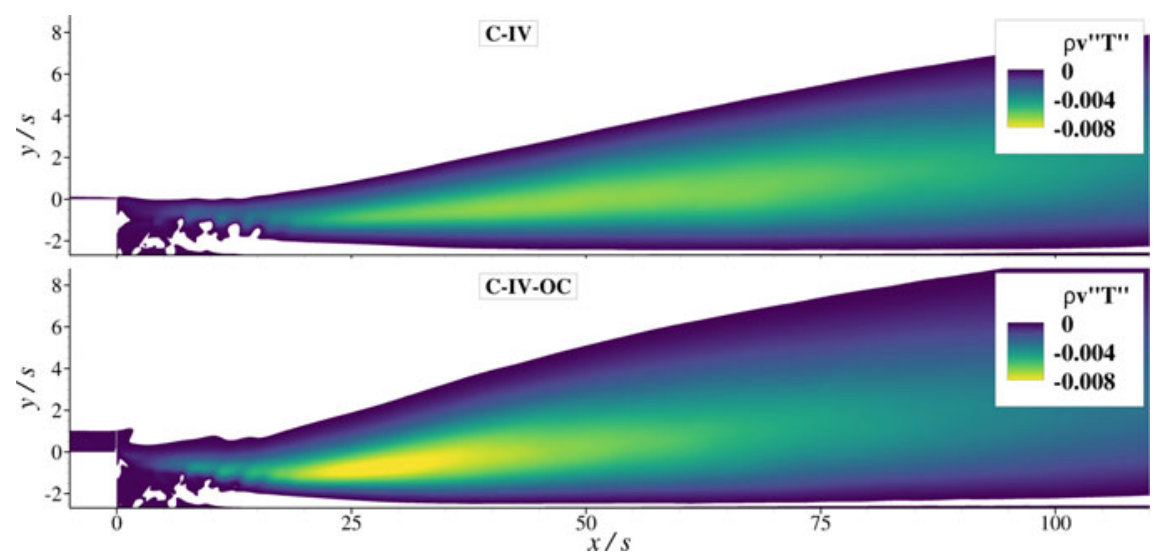

Fig. 7 Contours of the turbulent stress $\overline{\rho v^{\prime \prime} T^{\prime \prime}}$ (values $>0$ are blanked)

wall leads to an approximately $30 \%$ higher gradient $\partial \bar{u} / \partial y$ at the GIP, owing to the higher shear of the oncoming boundary layer. This leads to higher turbulence production in the vicinity of the step, causing increased mixing between the two gases and a higher transport of heat towards the wall due to turbulence, as can be seen from the (negative) turbulent heat flux contours in Fig. 7.

\subsection{Lip-Thickness Influence}

The lip thickness is an important dimension for the structural design of a filmcooling device. Several studies have shown a large impact of the lip thickness in subsonic flow [26], but only few studies have investigated the issue under super- or hypersonic conditions $[4,17,24]$. The effect of a change in lip thickness at kept slot height is investigated by comparing the cases listed in Table 6. A reduction of the lip thickness has virtually no effect on film cooling, increasing the lip thickness leads to slightly higher mixing and thus a (very) small reduction in downstream cooling, see Fig. 8. Overall, the influence is negligible and the data indicate that, within reasonable structural dimensions, the lip thickness can be determined by structural design constraints, in agreement with the experimental results by Olsen et al. [17]. Further investigations will show if this holds for matched-pressure and over-expanded coolant blowing. 
Table 6 Investigated cases for the lip-thickness ( $t *)$ influence

\begin{tabular}{l|l|l|l|l|l}
\hline Case & $F$ & Geometry & $s^{\star}(\mathrm{mm} 0$ & $t^{\star}(\mathrm{mm})$ & $\begin{array}{l}\text { Step height } \\
s^{\star}+t^{\star}(\mathrm{mm})\end{array}$ \\
\hline C-IV & 1.00 & G01 & 0.6 & 1.0 & 1.6 \\
\hline C-IV-t.2 & 1.00 & G01a & 0.6 & 2.0 & 2.6 \\
\hline C-IV-t/2 & 1.00 & G01b & 0.6 & 0.5 & 1.1 \\
\hline
\end{tabular}

Fig. 8 Distribution of $\eta_{a d}$ for different lip thicknesses $t$

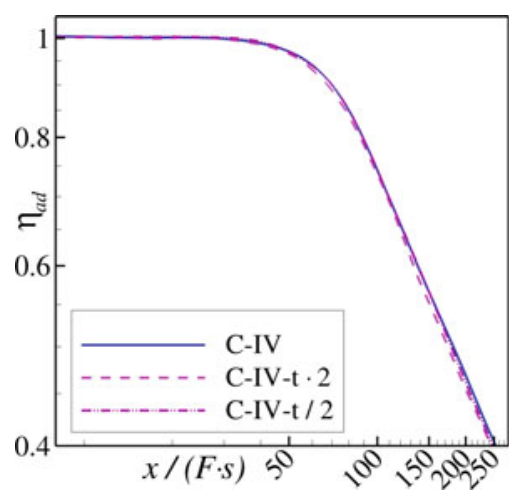

\subsection{Influence of the Coolant Velocity Profile}

The assumption of a parabolic velocity profile $u_{c}=f\left(y^{2}\right)$ at the coolant slot opening is rather generic, stemming from the assumption of the coolant supply resembling a laminar channel flow. In a practical application the stream profile would depend on the channel geometry and might not even be symmetrical. To assess a possible influence of this assumption the baseline case C-IV was modified to $u_{c}=f\left(y^{6}\right)$, see Table 7. This fuller velocity profile leads to a reduction in pressure for the same blowing ratio, but the flow is still under-expanded. Additionally, the velocity ratio $V=u_{c} / u_{\infty}$, and thus also the momentum ratio, increases. Figure 9 shows no major difference in the cooling effectiveness for both inlet profiles. The length of the potential-core region is virtually unchanged and the decay rate is only marginally increased. The fuller velocity profile leads to a higher shear rate on the coolant side of the mixing region, causing the slightly increased decay rate. Ultimately, the development of the mixing layer appears to be largely dominated by the main-flow free shear layer emanating at the upper edge of the lip, as the total shear stress (i.e. mean flow stress $\overline{\mu \cdot \partial u / \partial y}$ plus mean turbulent stress $\overline{\rho u^{\prime \prime} v^{\prime \prime}}$ ) here is much higher than in the coolant shear layer. Note that this might be different if the film cooling flow is not core driven, i.e. $V>1$. 
Table 7 Investigated cases for the coolant velocity-profile influence

\begin{tabular}{l|l|l|l|l|l|l}
\hline Case & $F$ & Geometry & $p_{c}^{\star}(\mathrm{Pa})$ & $V$ & $M$ & $\begin{array}{l}\text { Coolant velocity } \\
\text { profile }\end{array}$ \\
\hline C-IV & 1.00 & G01 & 47745 & 0.263 & 0.331 & $u_{c}=f\left(y^{2}\right)$ \\
\hline C-IV- $y^{6}$ & 1.00 & G01 & 31606 & 0.338 & 0.372 & $u_{c}=f\left(y^{6}\right)$ \\
\hline
\end{tabular}

Fig. 9 Distribution of $\eta_{a d}$ for a different coolant inlet velocity profiles

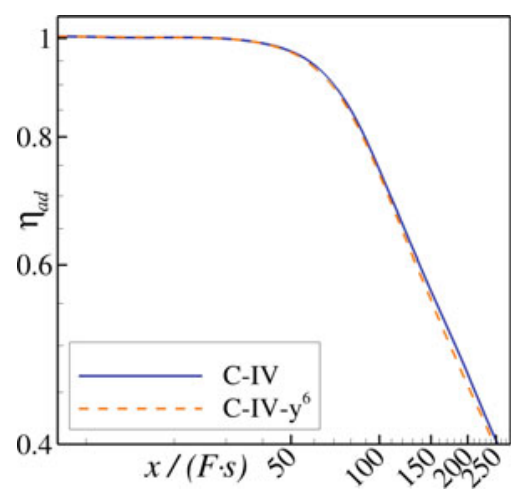

\subsection{Correlation of Data}

The correlation of film-cooling data is an important step in both experimental and numerical investigations, as it provides a valuable tool for the practical design-phase of a cooling system. Goldstein's mixing model [2]

$$
\eta_{a d}=\left[1+\frac{c_{p, \infty}}{c_{p, c}} \frac{\dot{m}_{\infty}}{\dot{m}_{c}}\right]^{-1}=\left[1+\frac{c_{p, \infty}}{c_{p, c}} \xi\right]^{-1},
$$

derived from a simple mass and energy balance in the boundary layer, is often used. Using a 1/7-th power law for the velocity profile and assuming the boundary-layer growth starts at the step, the correlation factor is given by

$$
\xi=\frac{7 \delta(x)}{8 F s}
$$

where $\delta(x)$ is the local boundary layer thickness, here derived from $\delta=0.37 x / R e_{x}^{0.2}$. Figure 10 shows the correlated cooling effectiveness for various cases as well as the modelled curve using Eq. 2. Also shown is a modified curve

$$
\eta_{a d, f i t}=\left[1+0.1101\left(\frac{c_{p, \infty}}{c_{p, c}} \xi\right)^{1.3934}\right]^{-1}
$$


using a data fit from the experimental results from sub-project "Film Cooling in Rocket Nozzle Flows". While the scaling factor $\xi$ does not correlate the DNS data very well, the fitted model shows much better agreement than the original variant. Note that the fitted curve matches the pre-cooled case C-IV-OC better than case C-IV. The results show that a relatively fast effectiveness loss near the injection rises with increasing upstream-wall pre-cooling.

\subsubsection{Comparison with Wall-Normal Blowing}

Figure 10 also shows DNS results for wall-normal slit blowing (WNB) into a turbulent air boundary layer, gained in the previous study campaign [10]. Comparing the helium injection data shows that the shown, specific-heat corrected, and thus fluiddynamical performance of WNB provides about the same cooling effectiveness than tangential blowing downstream, but close to the coolant injection, the tangential blowing is clearly superior due to its high streamwise momentum. Note that the turbulent kinetic energy of the mean flow can infiltrate the WNB channel, and also the mixing by temperature fluctuations is relatively high near the injection, see [10]. WNB with air instead of helium provides a higher corrected performance because the wall-normal blowing velocity is much smaller for a given blowing ratio due to the higher density, and thus the coolant stays closer at the wall. This means that WNB using a light, high heat-capacity gas is generally better than using a heavier gas concerning the absolute cooling and its effectiveness at kept blowing ratio, but some fluid-dynamical performance losses due to the higher WNB velocity go with it.

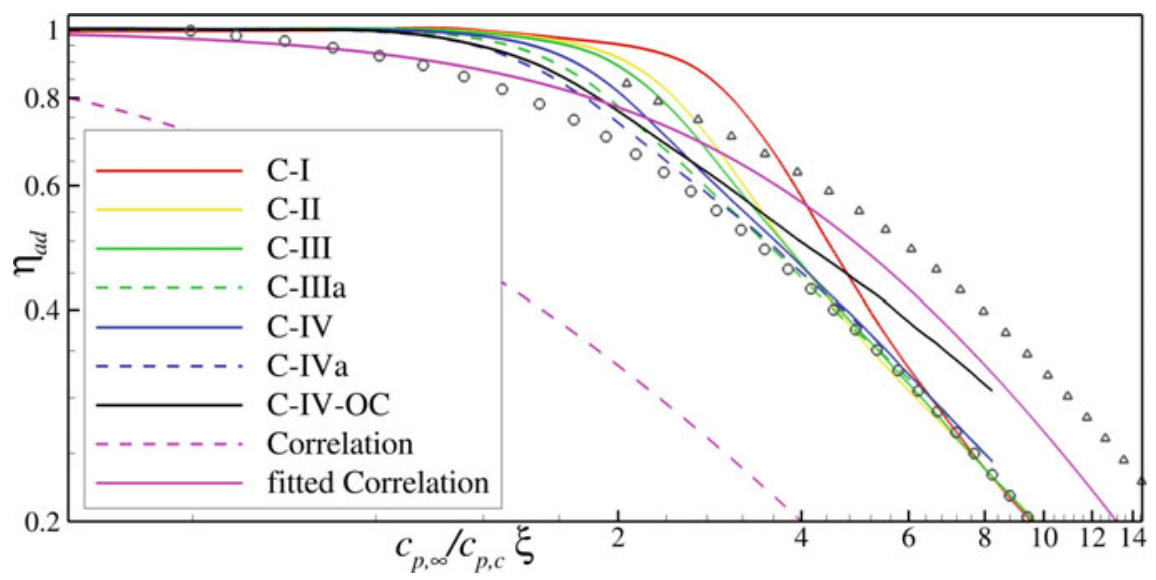

Fig. 10 Scaling of film cooling data. Correlation is Eq. 2, fitted correlation is Eq. 4. Symbols indicate data from [10] for wall-normal blowing with helium (circles) and air (triangles) 


\section{Conclusions and Outlook}

High-order DNS of film cooling by tangential blowing have been performed. The main flow is a turbulent boundary layer of hot steam at Mach 3.3, and cold helium is injected at supersonic speed. The coolant mass flow rate has been varied by varying the blowing ratio $F$ and the slot height $s$. Analysis of the adiabatic cooling effectiveness $\eta_{a d}$ shows the expected better performance for higher mass flow rate values, but the mass effectiveness $x /(F s)$ in the near-slot region is higher for lower blowing ratios. Additionally, injecting a kept mass flow rate $F \cdot s$ is more effective with a smaller slot height $s$. The coolant Mach number appears to have no significant influence on the flow mixing, as well as the lip thickness. Cooling the wall upstream of the blowing leads to a significantly higher shear and thus to a stronger turbulence production in the free shear layer behind the step. This leads to increased gas mixing as well as a higher turbulent transport of thermal energy towards the lower wall. Close to the slot the cooling effectiveness therefore shows a reduction compared to an adiabatic upstream wall - in accordance with experimental results, and only in the far downstream region the pre-cooling leads to the expected lower wall temperature.

The DNS results suggest that for any comprehensive scaling formula the effect of a non-adiabatic-wall incoming boundary-layer needs to be incorporated, either through additional factors in the correlation and/or non-constant parameters. This might prove to be a difficult task, and it remains questionable if an all-embracing scaling is possible. Existing correlation formulas (derived from experiments) might only be applicable to flow conditions for which they were derived, and caution is advised when applied for different setups.

The comparison of tangential with wall-normal blowing shows that the former has a higher effectiveness in the scaled region not far from the injection due to the high streamwise momentum that can be applied. More downstream, the scaled effectiveness-values conform. Of course, the typical, high blowing ratio of order 1 with tangential injection provides a substantially larger not-scaled, actual stretch of cooled wall with only one injection slot.

Next steps will comprise the evaluation of the wall-temperature and pressuregradient influence in cooling-effectiveness correlations and the analysis of turbulencemodelling parameters, e.g. the turbulent Prandtl and Schmidt numbers, from the gathered data. Comparisons of DNS results with selected RANS simulations will provide assistance for modelling of film cooling for industrial purposes.

Acknowledgements Financial support has been provided by the German Research Foundation (Deutsche Forschungsgemeinschaft - DFG) in the framework of the Sonderforschungsbereich Transregio 40 (SFB-TRR40, SP A4). The simulations were performed on the national supercomputer Cray XC40 'Hazelhen' at the High Performance Computing Center Stuttgart (HLRS) under grant GCS_Lamt, ID 44026. 


\section{References}

1. Christopher, N., Peter, J.M.F., Kloker, M.J., Hickey, J.P.: DNS of turbulent flat-plate flow with transpiration cooling. Int. J. Heat Mass Transf. 157 (2020) 119972. https://doi.org/10.1016/j. ijheatmasstransfer.2020.119972

2. Goldstein, R.J.: Film cooling. Adv. Heat Transf. 7, 321-379 (1971). https://doi.org/10.1016/ S0065-2717(08)70020-0

3. Goldstein, R.J., Eckert, E.R.G., Wilson, D.J.: Film cooling with normal injection into a supersonic flow. J. Eng. Ind. 90(4), 584-588 (1968). https://doi.org/10.1115/1.3604692

4. Holden, M.S., Rodriguez, K.: Experimental studies of shock-wave/wall-jet interaction in hypersonic flow. Tech Report NASA-CR-195844, Calspan-UB (1994)

5. Hombsch, M., Olivier, H.: Film cooling in laminar and turbulent supersonic flows. J. Spacecr. Rocket. 50(4), 742-753 (2013). https://doi.org/10.2514/1.a32346

6. Juhany, K.A., Hunt, M.L.: Flowfield measurements in supersonic film cooling including the effect of shock-wave interaction. AIAA J. 32(3), 578-585 (1994). https://doi.org/10.2514/3. 12024

7. Keller, M.: Numerical investigation of gaseous film and effusion cooling in supersonic boundary-layer flows. Ph.D. thesis, Universität Stuttgart (2016)

8. Keller, M., Kloker, M.J.: DNS of effusion cooling in a supersonic boundary-layer flow: influence of turbulence. In: 44th AIAA Thermophysics Conference (2013). https://doi.org/10.2514/6. 2013-2897. AIAA-2013-2897

9. Keller, M., Kloker, M.J.: Effusion cooling and flow tripping in laminar supersonic boundarylayer flow. AIAA J. 53(4) (2015). https://doi.org/10.2514/1.J053251

10. Keller, M., Kloker, M.J.: Direct numerical simulation of foreign-gas film cooling in supersonic boundary-layer flow. AIAA J. 55(1), 99-111 (2016). https://doi.org/10.2514/1.J055115

11. Keller, M., Kloker, M.J., Olivier, H.: Influence of cooling-gas properties on film-cooling effectiveness in supersonic flow. J. Spacecr. Rocket. 52(5), 1443-1455 (2015). https://doi.org/10. 2514/1.A33203

12. Klein, M., Sadiki, A., Janicka, J.: A digital filter based generation of inflow data for spatially developing direct numerical or large eddy simulations. J. Comput. Phys. 186(2), 652-665 (2003). https://doi.org/10.1016/s0021-9991(03)00090-1

13. Konopka, M., Meinke, M., Schröder, W.: Large-eddy simulation of shock/cooling-film interaction. AIAA J. 50(10), 2102-2114 (2012). https://doi.org/10.2514/1.J051405

14. Linn, J., Kloker, M.J.: Effects of wall-temperature conditions on effusion cooling in a supersonic boundary layer. AIAA J. 49(2), 299-307 (2011). https://doi.org/10.2514/1.J050383

15. Ludescher, S., Olivier, H.: Experimental investigations of film cooling in a conical nozzle under rocket-engine-like flow conditions. AIAA J. 57(3), 1172-1183 (2018). https://doi.org/ 10.2514/1.j057486

16. Mack, L.M.: Boundary-layer linear stability theory. Special Course on Stability and Transition of Laminar Flow - AGARD-R-709. AGARD (1984)

17. Olsen, G., Nowak, R., Holden, M., Baker, N.: Experimental results for film cooling in 2-D supersonic flow including coolant delivery pressure, geometry, and incident shock effects. In: 28th Aerospace Sciences Meeting (1990). https://doi.org/10.2514/6.1990-605

18. Peter, J.M.F., Kloker, M.J.: Preliminary work for DNS of rocket-nozzle film-cooling. Deutscher Luft- und Raumfahrtkongress DLRK, DLRK-2017-450178 (2017). http://d-nb.info/ 1142014584. Or see www.ResearchGate.net

19. Peter, J.M.F., Kloker, M.J.: Influence of upstream wall temperature on film cooling by tangential blowing. Technical Report, IAG USTUTT (2019). https://doi.org/10.13140/RG.2.2.15075. 50724

20. Peter, J.M.F., Kloker, M.J.: Direct numerical simulation of supersonic film cooling by tangential blowing. In: Nagel, W.E., Kröner, D.B., Resch, M.M. (eds.) High Performance Computing in Science and Engineering' 19. Springer International Publishing, Berlin (2020) 
21. Poggie, J., Bisek, N.J., Gosse, R.: Resolution effects in compressible, turbulent boundary layer simulations. Comput. Fluids 120, 57-69 (2015). https://doi.org/10.1016/j.compfluid.2015.07. 015

22. Song, C., Shen, C.: Effects of feeding pressures on the flowfield structures of supersonic film cooling. J. Thermophys. Heat Transf. 32(3), 648-658 (2018). https://doi.org/10.2514/1.t5322

23. Song, C., Shen, C.: Effects of feeding Mach numbers on the flowfield structures of supersonic film cooling. J. Thermophys. Heat Transf. 33(1), 264-270 (2019). https://doi.org/10.2514/1. t5475

24. Song, C., Shen, C.: Effects of lip thickness on the flowfield structures of supersonic film cooling. J. Thermophys. Heat Transf. 33(3), 599-605 (2019). https://doi.org/10.2514/1.t5479

25. Stollery, J.L., El-Ehwany, A.A.M.: A note on the use of a boundary-layer model for correlating film-cooling data. Int. J. Heat Mass Transf. 8(1), 55-65 (1965). https://doi.org/10.1016/00179310(65)90097-9

26. Taslim, M.E., Spring, S.D., Mehlman, B.P.: Experimental investigation of film cooling effectiveness for slots of various exit geometries. J. Thermophys. Heat Transf. 6(2), 302-307 (1992). https://doi.org/10.2514/3.359

27. Wenzel, C., Selent, B., Kloker, M.J., Rist, U.: DNS of compressible turbulent boundary layers and assessment of data-/scaling-law quality. J. Fluid Mech. 842, 428-468 (2018). https://doi. org/10.1017/jfm.2018.179

Open Access This chapter is licensed under the terms of the Creative Commons Attribution 4.0 International License (http://creativecommons.org/licenses/by/4.0/), which permits use, sharing, adaptation, distribution and reproduction in any medium or format, as long as you give appropriate credit to the original author(s) and the source, provide a link to the Creative Commons license and indicate if changes were made.

The images or other third party material in this chapter are included in the chapter's Creative Commons license, unless indicated otherwise in a credit line to the material. If material is not included in the chapter's Creative Commons license and your intended use is not permitted by statutory regulation or exceeds the permitted use, you will need to obtain permission directly from the copyright holder.

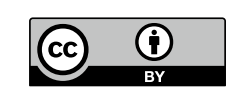

